\title{
Online Effective Identification of Glycopeptide Using Liquid Chromatography Combined with Fourier Transform Ion Cyclotron Resonance Mass Spectrometry (FTICR-MS)*
}

\author{
Ling Xin, Li Hou, Yue Zhou, Huiping Wang, Heming Yu \\ National Health and Family Planning Key Laboratory of Male Reproductive Health, Department of Reproductive \\ Immunology, National Research Institute for Health and Family Planning, Beijing, China \\ Email: xinling_w1225@163.com
}

Received 29 June 2015; accepted 21 September 2015; published 24 September 2015

Copyright (C) 2015 by authors and Scientific Research Publishing Inc.

This work is licensed under the Creative Commons Attribution International License (CC BY).

http://creativecommons.org/licenses/by/4.0/

\section{(c) (i) Open Access}

\begin{abstract}
The detailed glycan structural analysis of glycoprotein is amenable to glycopeptide enrichment. Here, we develop a simple, effective and economical approach to enrich glycopeptides from proteolytically digested peptide mixtures by chromatographic column packed with graphite carbon and activated charcoal (G/A-column). Glycopeptide from ovalbumin was efficiently enriched by homemade G/A-column using liquid chromatography and the structure of glycopeptide was obtained by tandem mass spectrometry using Fourier transform ion cyclotron resonance mass spectrometry. The results in this study demonstrate that G/A-column can be used to enrich N-glycolpeptides and be benefit for online identification of glycopeptide using LC-MS.
\end{abstract}

\section{Keywords}

Glycopeptide, LC-ESI, FTICR-MS

\section{Introduction}

Various investigations concerning oligosaccharide analysis have been performed because of the importance of

*Identification of glycopeptide using LC-MS.

How to cite this paper: Xin, L., Hou, L., Zhou, Y., Wang, H.P. and Yu, H.M. (2015) Online Effective Identification of Glycopeptide Using Liquid Chromatography Combined with Fourier Transform lon Cyclotron Resonance Mass Spectrometry (FTICRMS). Advances in Bioscience and Biotechnology, 6, 624-628. http://dx.doi.org/10.4236/abb.2015.69065 
oligosaccharide moiety in glycoprotein in biological systems [1] [2]. Intact glycopeptide can provide structural information of glycans as well as peptide, which includes the information of glycosylation site. Mass spectrometry (MS) is one of the most powerful and versatile techniques for identification of glycopeptides [3] [4]. However, due to low abundance of glycopeptides, low MS signal was suppressed by non-glycopeptides. It is a real demand to separate glycopeptides for the detailed glycopeptide structural analysis. Liquid chromatography (LC) combined with MS can hit the mark that isolates and identifies glycopeptides online [5] [6].

A number of experiments for enrichment of glycopeptides using graphite carbon and activated charcoal, which has been mostly used in pharm, have been reported [7] [8]. Based on the similar properties, it is regarded that there are differences in adsorptions, signal intensity, $\mathrm{S} / \mathrm{N}$ ratio, peptide binding and loading capacity between graphite carbon and activated charcoal [9] [10].

Here, we develop a relatively stable, effective and economical approach to isolate and identify glycopeptides using liquid chromatography (LC) online combined with electrospray ionization Fourier transform ion cyclotron resonance (FTICR) mass spectrometry (MS). Characterization of glycopeptides derived from ovalbumin was analyzed. The result demonstrated that G/A-column could efficiently isolate the glycopeptides from the digestion mixtures of ovalbumin, and detailed glycan structural analysis was obtained by using tandem mass spectrometry.

\section{Experimental}

\subsection{Materials and Chemicals.}

Graphite carbon powder was from Alltech (Sydney, Australia). Activated charcoal, ovalbumin, dithiothreitol (DTT), iodoacetamide, proteinase K were from Sigma (St. Louis, MO). Trypsin (sequencing-grade modified) was purchased from Promega (Madison, WI). The ddwater was from a Milli-Q system (Millipore, Bedford, MA). The other reagents were chromatographic-grade.

\subsection{In-Gel Digest and Proteinase $\mathrm{K}$ Digest.}

After resolved on 12\% SDS-PAGE [11], in-gel digestion was performed as the protocol [12] with modification fallowed by proteinase $\mathrm{K}$ digestion. The supernatant from the tryptic digestion of the ovalbumin was transferred to a new tube. Proteinase $\mathrm{K}$ dissolved in water $(1 \mathrm{mg} / \mathrm{mL})$ was added to the tube, which the final concentration of proteinase $\mathrm{K}$ was up to $0.1 \mu \mathrm{g} / \mu \mathrm{L}$. The digestion was carried out at $37^{\circ} \mathrm{C}$ overnight. The solution of digestion was dried by Speedvac and dissolved in $1 \%$ formic acid to optimize the $\mathrm{pH}$ condition facilitating the glycopeptide binding to packing materials.

\subsection{Liquid Chromatography-Mass Spectrometry.}

Homemade columns $(1000 \mu \mathrm{m} \times 30 \mathrm{~mm})$ were packed with a acetonitrile slurry of graphite carbon and activated charcoal particles (w/w, 1:1) at $<1500$ psi. Samples digested by trypsin and proteinase $\mathrm{K}$ in-gel were injected in $0.2 \%$ formic acid in water onto the homemade column coupled to a Bruker Apex Ultra FTICR mass spectrometer (Bruker Daltonics, Germany) for tandem MS analysis. Mobile phase A was $0.2 \%$ formic acid in water. Mobile phase B was $0.2 \%$ formic acid in acetonitrile. Chromatography was performed at a flow rate of $5 \mu \mathrm{L} / \mathrm{min}$ with a ladder gradient of $0 \%, 20 \%, 30 \%$ B in 60 min following a linear gradient of $30 \%-90 \%$ B in 20 min while holding at $95 \%$ B for $10 \mathrm{~min}$.

All MS experiments were carried out in positive ion mode, the electrospray conditions were optimized as spray voltage $\mathrm{kV}$ and capillary temperature $150^{\circ} \mathrm{C}$. PepSeq was used along with ExPASy's GlycoMod Tool to predict potential glycan compositions from glycan masses determined from MS/MS analysis of intact glycopeptides [13].

\section{Results and Discussion}

Glycosylation is one of the most common post-translational modifications (PTMs). It is important to isolate and identify subsequently glycosylated peptides in glycoproteomics [14] [15]. LC-MS is one of the most powerful and versatile techniques for isolation and identification of glycopeptides. 
Some experiments for analysis of glycopeptides or oligosaccharide by graphite carbon combining LC-MS have been performed [7]. Activated charcoal also has been used to trap glycopeptides due to its adsorption [10]. Based on the difference in physicochemical properties, the columns were packed with graphite carbon and activated charcoal in equal weight (w/w, 1:1).

Ovalbumin is a well-characterized glycoprotein containing a N-linked glycosylation site (Asn292). Proteinase $\mathrm{K}$ is a nonspecific proteinase, which can cleave the majority of the tryptic peptides into smaller peptides consisting of three to five amino acid residues. After digestion by trypsin and proteinase K, small glycopeptides that will have molecular masses typically $>1200 \mathrm{Da}$. The mass spectrum acquired by infusing an enzymatic mixture of ovalbumin onto the G/A homemade column is presented in Figure 1. The results show that the peak of glycopeptide arises at thirty-fourth to fortieth minute (Figure 1(a)). ESI produced doubly protonated ions possessing $\mathrm{m} / \mathrm{z}$ values of $944.9(2+), 986.4(2+), 1067.5(2+), 1087.5(2+), 1168.5(2+)$ and 1249.6(2+), respectively (Figure 1(b)), which indicate the mass of glycopeptides is 1889.8 Da, 1972.8 Da, 2135.1 Da, 2175.0 Da, 2337.0 $\mathrm{Da}$, and $2499.2 \mathrm{Da}$. It is shown that the mass gaps between peaks correspond to the typical monosaccharide masses (162 and 203 Da for hexose and N-acetyl-hexosamine, respectively), suggesting the presence of a peptide with a heterogenous glycan structures.

The glycopeptide structures in ovalbumin can be identified by tandem mass spectrometry. The glycopeptide mass is selected in the quadrupole and submitted to collision-induced dissociation (CID). Figure 2 shows the fragment ion spectra obtained from the glycopeptide of ovalbumin. It is shown that glycopeptide derived from ovalbumin are splitted to smaller glycopeptide and a series of glycan. Based on the collision so the significant peaks at $\mathrm{m} / \mathrm{z}$ 944.9(2+), 986.4(2+), 1067.5(2+), 1087.5(2+), 1168.5(2+) and 1249.6(2+) observed in Figure 1(b) correspond to the same peptide sequence (YNLT) carrying (GlcNAc) ${ }_{2} \mathrm{Man}_{3} \mathrm{Hex}_{3},(\mathrm{GlcNAc})_{2} \mathrm{Man}_{3}\left(\mathrm{HexNAc}_{2} \mathrm{Hex}_{1}\right.$, $(\text { GlcNAc })_{2} \mathrm{Man}_{3}(\mathrm{HexNAc})_{2} \mathrm{Hex}_{2}$, (GlcNAc) $\mathrm{Man}_{3}\left(\mathrm{HexNAc}_{3} \mathrm{Hex}_{1},(\mathrm{GlcNAc})_{2} \mathrm{Man}_{3}\left(\mathrm{HexNAc}_{3} \mathrm{Hex}_{2}\right.\right.$, (GlcNAc) ${ }_{2} \mathrm{Man}_{3}(\mathrm{HexNAc})_{3} \mathrm{Hex}_{3}$, respectively. The characterization of glycopeptide of ovalbumin can be obtained.

Graphite carbon and activated charcoal may preferably target different interacting interfaces in single glycolpeptides, which results in complementary absorptions. Glycoprotein produces small glycopeptides that contain

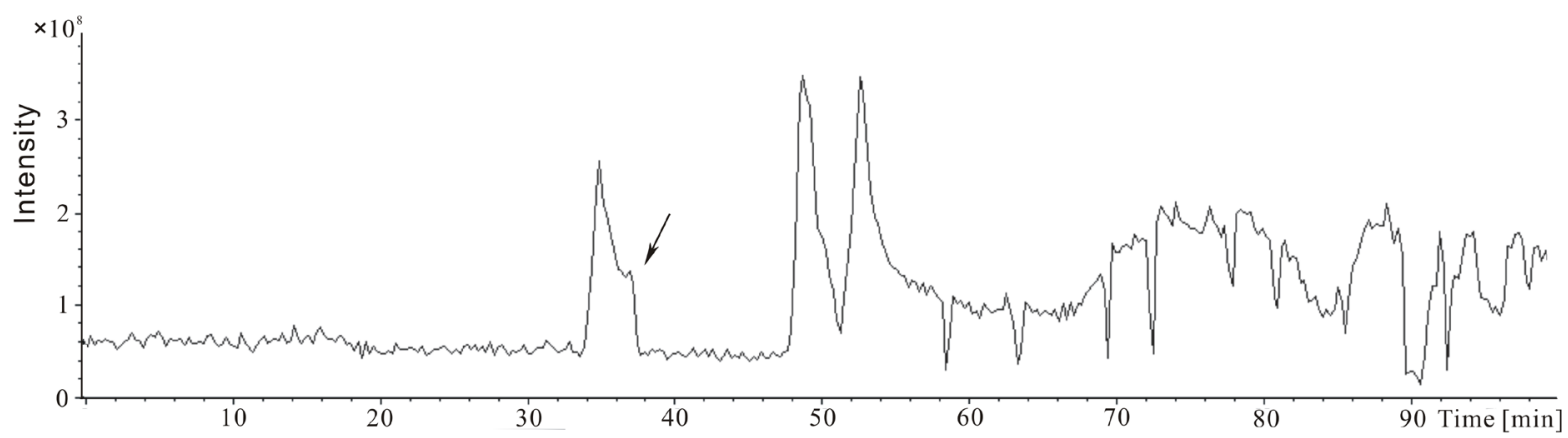

(a)

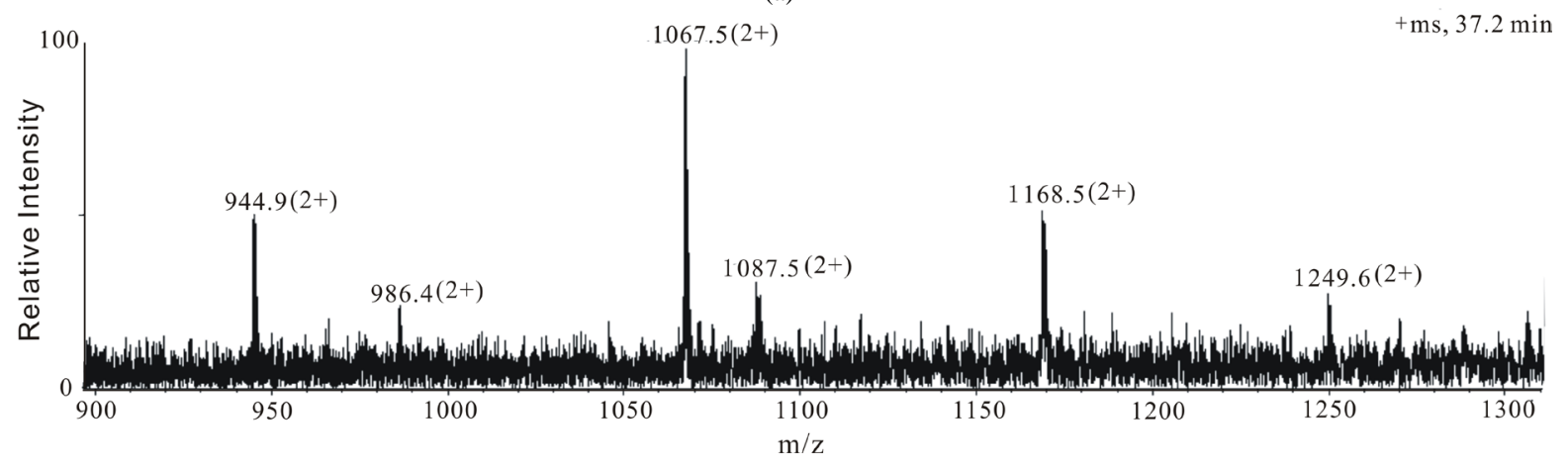

(b)

Figure 1. LC-ESI mass spectrum of ovalbumin. (a) Total ion chromatogram (TIC) of digestion from ovalbumin; (b) Positive-ion ESI mass spectrum of glycopeptide derived from ovalbumin, which was collected at 37.2 minute indicated by arrow. 


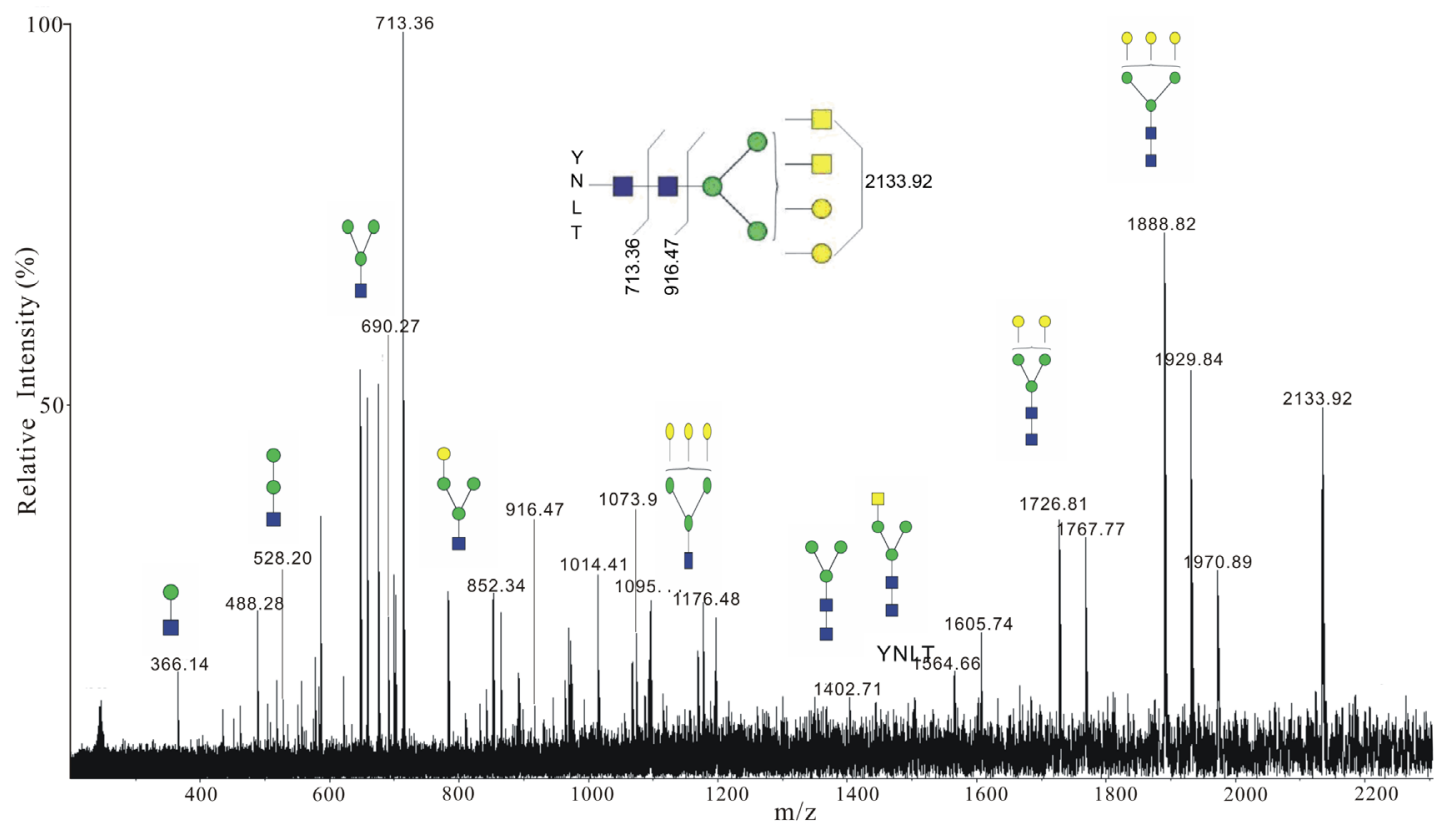

Figure 2. Fragmentation of glycopeptides from ovalbumin. The $m / z$ of precursor ion was 2133.92, which was the peak at $m / z$ 1067.5(2+) in Figure 1(b). Blue quadrate indicates N-acetylglucosamine (GlcNAc); Green circle indicates mammose (Man); Yellow quadrate indicates $\mathrm{N}$-acetylhexosamine (HexNAc); Yellow circle indicates hexose (Hex).

a peptide tag by two-stage digestion using trypsin and proteinase $\mathrm{K}$. These glycopeptides resemble free glycans with respect to hydrophilicity. Due to glycan structures in general are highly hydrophilic they can be separated by using normal-phase liquid chromatography.

\section{Conclusion}

In this study, a series of glycopeptide signal peaks derived from digestion of ovalbumin are detected, showing that the G/A-column has the potential to isolate and enrich glycopeptides specifically and efficiently. By using Tandem mass spectrometry, the characterizations of glycopeptides derived from ovalbumin were obtained. Therefore it will be benefit for glycoproteomics. Moreover our strategy is simple and economic for glycopeptides analysis.

\section{References}

[1] Min, J.Z., Kurihara, T., Hirata, A., Toyo’oka, T. and Inagaki, S. (2009) Identification of N-Linked Oligosaccharide Labeled with 1-Pyrenesulfonyl Chloride by Quadrupole Time-of-Flight Tandem Mass Spectrometry after Separation by Micro- and Nanoflow Liquid Chromatography. Biomedical Chromatography, 23, 912-921. http://dx.doi.org/10.1002/bmc.1201

[2] Kurihara, T., Min, J.Z., Hirata, A., Toyo’oka, T. and Inagaki, S. (2009) Rapid Analysis of N-Linked Oligosaccharides in Glycoproteins (Ovalbumin, Ribonuclease B and Fetuin) by Reversed-Phase Ultra-Performance Liquid Chromatography with Fluorescence Detection and Electrospray Ionization Time-of-Flight Mass Spectrometry. Biomedical Chromatography, 23, 516-523. http://dx.doi.org/10.1002/bmc.1147

[3] Harvey, D.J. (2005) Proteomic Analysis of Glycosylation: Structural Determination of N- and O-Linked Glycans by Mass Spectrometry. Expert Review of Proteomics, 2, 87-101. http://dx.doi.org/10.1586/14789450.2.1.87

[4] Morelle, W., Faid, V., Chirat, F. and Michalski, J.C. (2009) Analysis of N- and O-Linked Glycans from Glycoproteins Using MALDI-TOF Mass Spectrometry. Methods in Molecular Biology, 534, 5-21. http://dx.doi.org/10.1007/978-1-59745-022-5_1

[5] Kawasaki, N., Toh, S., Ohta, M. and Hayakawa, T. (2003) Microanalysis of N-Linked Oligosaccharides in a Glycoprotein by Capillary Liquid Chromatography/Mass Spectrometry and Liquid Chromatography/Tandem Mass Spectrometry. Analytical Biochemistry, 316, 15-22. http://dx.doi.org/10.1016/S0003-2697(03)00031-9

[6] Itoh, S., Kawasaki, N., Hashii, N., Harazono, A., Matsuishi, Y., Hayakawa, T. and Kawanishi, T. (2005) N-Linked 
Oligosaccharide Analysis of Rat Brain Thy-1 by Liquid Chromatography with Graphitized Carbon Column/Ion TrapFourier Transform Ion Cyclotron Resonance Mass Spectrometry in Positive and Negative Ion Modes. Journal of Chromatography A, 1103, 296-306. http://dx.doi.org/10.1016/j.chroma.2005.11.043

[7] Ruhaak, L.R., Deelder, A.M. and Wuhrer, M. (2009) Oligosaccharide Analysis by Graphitized Carbon Liquid Chromatography-Mass Spectrometry. Analytical and Bioanalytical Chemistry, 394, 163-174. http://dx.doi.org/10.1007/s00216-009-2664-5

[8] El Bahri, L. (2008) Pharm Profile: Activated Charcoal. Compendium on Continuing Education for the Practising Veterinarian, 30, 596-598.

[9] Larsen, M.R., Hojrup, P. and Roepstorff, P. (2005) Characterization of Gel-Separated Glycoproteins Using Two-Step Proteolytic Digestion Combined with Sequential Microcolumns and Mass Spectrometry. Molecular \& Cellular Proteomics, 4, 107-119. http://dx.doi.org/10.1074/mcp.M400068-MCP200

[10] Larsen, M.R., Cordwell, S.J. and Roepstorff, P. (2002) Graphite Powder as an Alternative or Supplement to ReversedPhase Material for Desalting and Concentration of Peptide Mixtures Prior to Matrix-Assisted Laser Desorption/Ionization-Mass Spectrometry. Proteomics, 2, 1277-1287. http://dx.doi.org/10.1002/1615-9861(200209)2:9<1277::AID-PROT1277>3.0.CO;2-P

[11] Werhahn, W. and Braun, H.P. (2002) Biochemical Dissection of the Mitochondrial Proteome from Arabidopsis thaliana by Three-Dimensional Gel Electrophoresis. Electrophoresis, 23, 640-646. http://dx.doi.org/10.1002/1522-2683(200202)23:4<640::AID-ELPS640>3.0.CO;2-F

[12] Mortz, E., Vorm, O., Mann, M. and Roepstorff, P. (1994) Identification of Proteins in Polyacrylamide Gels by Mass Spectrometric Peptide Mapping Combined with Database Search. Biological Mass Spectrometry, 23, 249-261. http://dx.doi.org/10.1002/bms.1200230503

[13] Blake, T.A., Williams, T.L., Pirkle, J.L. and Barr, J.R. (2009) Targeted N-Linked Glycosylation Analysis of H5N1 Influenza Hemagglutinin by Selective Sample Preparation and Liquid Chromatography/Tandem Mass Spectrometry. Analytical Chemistry, 81, 3109-3118. http://dx.doi.org/10.1021/ac900095h

[14] Arnold, J.N., Wormald, M.R., Sim, R.B., Rudd, P.M. and Dwek, R. (2007) The Impact of Glycosylation on the Biological Function and Structure of Human Immunoglobulins. Annual Review of Immunology, 25, 21-50. http://dx.doi.org/10.1146/annurev.immunol.25.022106.141702

[15] Yu, L., Li, X., Guo, Z., Zhang, X. and Liang, X. (2009) Hydrophilic Interaction Chromatography Based Enrichment of Glycopeptides by Using Click Maltose: A Matrix with High Selectivity and Glycosylation Heterogeneity Coverage. Chemistry, 15, 12618-12626. http://dx.doi.org/10.1002/chem.200902370 